\title{
Hábitos lectores y competencia léxica de futuros profesores de inglés en Educación Primaria
}

\author{
Guadalupe de la Maya Retamari \\ Universidad de Extremadura, Badajoz, España \\ Magdalena López-Pérez ${ }^{\mathrm{ii}}$ iD \\ Universidad de Extremadura, Badajoz, España
}

\begin{abstract}
Resumen
Este artículo pretende reflexionar sobre la competencia lingüística y el desarrollo de hábitos lectores en la educación superior, centrándose específicamente en el análisis de la competencia léxica de los futuros profesores de inglés de Primaria y sus usos lectores en esta lengua extranjera. En la investigación han participado 34 estudiantes del Itinerario de intensificación de inglés, del Grado de Educación Primaria de la Facultad de Educación de la Universidad de Extremadura. Dichos estudiantes completaron un cuestionario para determinar la extensión de su vocabulario receptivo y un sencillo test en el que informaban de sus hábitos de lectura. La mayoría de los alumnos no tiene aún adquirido el conocimiento del léxico de las 3.000 palabras más frecuentes y tampoco tiene afianzado un hábito lector en lengua extranjera. Por último, en el análisis de correlación efectuado no se aprecia relación entre la frecuencia de lectura y el tamaño del vocabulario.
\end{abstract}

Palabras clave

Lectura. Vocabulario. Formación inicial del profesor. Lenguas extranjeras.

\section{Hábitos de leitura e competência lexical de futuros professores de inglês na Educação Primária}

\begin{abstract}
Resumo
Este artigo pretende refletir sobre a competência linguística e o desenvolvimento de hábitos de leitura no ensino superior, focando especificamente a análise da competência lexical de futuros professores primários de inglês e seus usos de leitura nessa língua estrangeira. Participaram da pesquisa 34 alunos do Itinerário de Intensificação de Inglês do Ensino Fundamental da Faculdade de Educação da Universidade de Extremadura. Esses alunos preencheram um questionário para determinar a extensão de seu vocabulário receptivo e um teste simples no qual relataram seus hábitos de leitura. A maioria dos alunos ainda não adquiriu o conhecimento do léxico das 3.000 palavras mais frequentes e também não estabeleceu um hábito de leitura em uma língua estrangeira. Por fim, na análise de correlação realizada, não foi observada relação entre frequência de leitura e tamanho do vocabulário.
\end{abstract}

\section{Palavras-chave}

Leitura. Vocabulário. Formação inicial do professor. Línguas estrangeiras.

Educ. Form., Fortaleza, v. 6, n. 1, e3504, jan./abr. 2021

DOI: https://doi.org/10.25053/redufor.v6i1.3504

https://revistas.uece.br/index.php/redufor/index ISSN: 2448-3583 


\title{
Reading habits and lexical competence of future teachers of English in Primary Education
}

\begin{abstract}
This article aims to reflect on linguistic competence and the development of reading habits in higher education, specifically focusing on the analysis of the lexical competence of future primary English teachers and their reading uses in this foreign language. 34 students from the English Intensification Itinerary, from the Primary Education Degree at the Faculty of Education from the University of Extremadura have participated in the research. These students completed a questionnaire to determine the extent of their receptive vocabulary and a simple test in which they reported their reading habits. Most of the students have not yet acquired the knowledge of the lexicon of the 3000 most frequent words and neither have they established a reading habit in a foreign language. Lastly, in the correlation analysis carried out, no relationship between reading frequency and vocabulary size was observed.
\end{abstract}

\section{Keywords}

Reading. Vocabulary. Initial teacher training. Foreign Languages.

\section{Introducción}

En el contexto español, la formación del profesorado de lenguas extranjeras en Educación Primaria ha sufrido una importante renovación con la entrada de nuestro país en el Espacio Europeo de Educación Superior. Así, una formación de tres cursos, especializada desde el primer año, ha dado paso a una formación generalista de un año más de duración, en la que la formación de los futuros profesores de inglés se concentra en el último año del grado, mediante el seguimiento de un itinerario de especialización que se articula en torno a tres o cuatro materias optativas. De este modo, se ha producido una reducción de la carga lectiva destinada a la especialización, coincidiendo, además, con un aumento de los posibles escenarios de actuación por la generalización de la enseñanza bilingüe, que demanda una formación especializada crucial para el éxito de estas experiencias innovadoras (BERTAUX et al., 2010; FRIGOLS et al., 2011; PAVÓN; ELLISON, 2013; PÉREZ CAÑADO, 2016).

Las competencias de los profesores de lenguas extranjeras son claves para una educación en idiomas de calidad. Como plantean desde el Centro Europeo de Lenguas Modernas, el aprendizaje exitoso de idiomas depende en gran medida del conocimiento, las habilidades y las actitudes de los profesores de idiomas. A este respecto existen 
numerosas propuestas que plantean cuáles deben ser esas competencias clave del profesorado (BLEICHENBACHER et al., 2016), esto es, qué conocimientos y habilidades necesita para ejercer su trabajo, tanto a nivel didáctico como lingüístico y cultural. Sin entrar en la determinación de niveles de dominio que, a veces, son planteados también en fases de desarrollo, lo que ponen en relieve estas propuestas es la exigencia de contar con profesionales que dominen convenientemente la lengua que deben enseñar, de ahí que este aspecto sea clave en los programas de formación inicial del profesorado. Partiendo de esta premisa, en este trabajo, pretendemos reflexionar sobre esa competencia lingüística del profesorado en formación y, más concretamente, sobre su competencia léxica y el desarrollo de hábitos lectores.

Más allá del rol central que la adquisición del vocabulario tiene en el aprendizaje de una lengua (BOGAARDS, 1994; LAUFER, 1986; WILKINS, 1974), existen numerosas contribuciones que respaldan la importancia del léxico en el uso de la lengua (SCHMITT, 2010), su condición de indicador de la competencia general que se posee en una determinada lengua, de modo que, cuanto mayor es el vocabulario, mayor es también la competencia (ALDERSON, 2005; FAN, 2000; HILTON, 2007; MEARA; JONES, 1988; MILTON, 2010; OVTCHAROV; COBB; HALTER, 2006; ZAREVA; SCHWANENFLUGEL; NIKOLOVA, 2005) y la relación que mantiene con las distintas destrezas, tales como la comprensión escrita, la expresión escrita o, en menor grado, la comprensión oral (AGUSTÍN LLACH; TERRAZAS GALLEGO, 2009; MILTON; WADE; HOPKINS, 2010; QIAN, 2002; STAEHR, 2008; WANG; TREFFERS-DALLER, 2017). Así, por ejemplo, Alderson (2005), en su investigación acerca del DIALANG, muestra cómo existe una relación significativa entre el léxico y la comprensión oral y escrita. Las conclusiones del estudio de Staehr (2008) arrojan este mismo resultado, corroborando que el aumento del léxico está fuertemente relacionado con el éxito en comprensión oral y escrita y también, aunque con un carácter menos intenso, con la comprensión oral. En la vertiente oral de la lengua, se centra precisamente el trabajo de Hilton (2008), que pone de manifiesto la relación entre el conocimiento léxico y la fluidez en una lengua, de modo que, cuantas más palabras se conocen, más fluidez se posee en el habla.

Como hemos señalado anteriormente, en el aprendizaje de una lengua, el vocabulario es un aspecto clave: 
No language acquisition, whether first, second, or foreign; child, or adult, can take place without the acquisition of lexis. Sound patterns of a language which do not form a lexical item are no more than meaningless noise; grammatical rules in themselves, unless they relate particular sounds to particular meanings, are only interesting abstractions with insufficient communicative value. (LAUFER, 1986, p. 69$)^{1}$.

Por ello, resulta evidente la importancia de que los alumnos posean un vocabulario amplio, lo que ha llevado a diferentes autores al establecimiento de umbrales léxicos para la realización de determinadas tareas comunicativas. Así, por lo que respecta a la comprensión de textos orales, el trabajo desarrollado por Adolphs y Schmitt (2004), estima en, al menos, 2000 palabras el vocabulario necesario para alcanzar una cobertura de entre el 90,36 \% y el 94,30\%, dependiendo del contexto del discurso oral. Van Zeeland y Schmitt (2013) cifran entre 2.000 y 3.000 familias el conocimiento necesario para alcanzar una cobertura del $95 \%$ que permitiría una adecuada comprensión oral. Por lo que respecta a la comprensión de textos escritos, Laufer (1989) concluye que una cobertura léxica del $95 \%$ puede asegurar una comprensión razonable, por lo que fija en 5.000 palabras o 3.000 familias de palabras (LAUFER, 1992) el umbral léxico para una comprensión satisfactoria. En un trabajo posterior, Laufer junto a Ravenhorst-Kalovski (2010) sugieren dos umbrales léxicos, uno mínimo y otro óptimo. El primero supone el conocimiento de 4.000-5.000 palabras y una cobertura del $95 \%$ y permite que los alumnos lean con alguna ayuda. El segundo, el umbral óptimo, pronostica una lectura independiente e implica el conocimiento de 8.000 familias de palabras que se traducen en una cobertura del $98 \%$. Como señalan Van Zeeland y Schmitt (2013), estas cifras de cobertura léxica son muy importantes, ya que nos permiten calcular el tamaño del vocabulario necesario para el uso de la lengua en tareas de comprensión oral o escrita.

La adquisición del vocabulario, como indica Schmitt (2010), es incremental en lo que se refiere tanto a la adquisición de un tamaño adecuado de vocabulario como al conocimiento individual de los ítems léxicos. No en vano, como señalaron Richards (1976) y Nation (2001), conocer una palabra supone el dominio de una serie de aspectos

\footnotetext{
1 "Ninguna adquisición de lenguas, ya sea primera, segunda o extranjera; por niños o adultos, puede ocurrir sin la adquisición del léxico. Los patrones de sonido de una lengua que no constituyen un elemento léxico no son más que sonidos sin sentido. Las reglas gramaticales en sí mismas, a menos que relacionen sonidos particulares con significados particulares, son únicamente interesantes abstracciones con un valor comunicativo insuficiente" (LAUFER, 1986, p. 69, traducción nuestra).
}

Educ. Form., Fortaleza, v. 6, n. 1, e3504, jan./abr. 2021

DOI: https://doi.org/10.25053/redufor.v6i1.3504

https://revistas.uece.br/index.php/redufor/index ISSN: 2448-3583 
(forma, significado y uso, en sus vertientes receptiva y productiva) que dan cuenta de la complejidad de esta tarea. Esta adquisición, además, difiere en el caso de la L1 y la L2, principalmente por la cantidad de input que, en un contexto y otro, recibe el alumno (WEBB; NATION, 2017). Asimismo, aunque generalmente pensamos en el aprendizaje concebido de una manera intencional, con una planificación por parte del profesor, la mayor parte del aprendizaje, en el caso de la L1, se hace de manera incidental (NATION, 2001). De nuevo, la cantidad de vocabulario aprendido por esta vía estará en dependencia de la cantidad de input y del número de veces que encontremos determinadas palabras, de modo que, a más contactos repetidos, más posibilidades de aprenderlas (WEBB; CHANG, 2015). En el caso de la L2, el escaso tiempo dedicado al aprendizaje de la lengua extranjera - y consecuentemente el input limitado que reciben los estudiantes -reduce, de un lado, el impacto de la frecuencia y del rango de aparición de las palabras en el aprendizaje del vocabulario (WEBB; CHANG, 2012) y, de otro, hace poco probable, como indican Webb y Nation (2017), que el estudiante pueda aprender todo el vocabulario necesario para realizar las tareas comunicativas enunciadas anteriormente, por lo que el aprendizaje incidental puede contribuir también al crecimiento del vocabulario, aunque de un modo mucho más limitado. No obstante, como plantean estos investigadores, es importante considerar los beneficios que proporcionan un tipo y otro de aprendizaje: más rápido y sustentado sobre la cantidad, el primero, y de más calidad y profundidad, el segundo, pues permite el aprendizaje de ciertos aspectos, como colocaciones (PELLICER-SÁNCHEZ, 2017), derivaciones o asociaciones. En este sentido, Restrepo Ramos (2015) señala que los estudiantes de una segunda lengua desarrollan la mayoría de su vocabulario incidentalmente por medio de la ocurrencia léxica en contextos altamente informativos, ocurrencia que se ve incentivada y complementada por la lectura y las anotaciones multimodales.

La investigación se ha interesado especialmente por el aprendizaje a través de la lectura (HORST; COBB; MEARA, 2008) y, más recientemente, también por este tipo de aprendizaje a partir de input de tipo audiovisual: programas de televisión en L2 (PETERS; WEBB, 2018; RODGERS; WEBB, 2019) o vídeos de corta duración (MONTERO-PEREZ, 2019; MONTERO-PEREZ et al., 2014), en los que, además de comprobarse que se efectúan ganancias de vocabulario, aunque estas se sitúan en etapas iniciales de

Educ. Form., Fortaleza, v. 6, n. 1, e3504, jan./abr. 2021 
aprendizaje, se evalúan también determinadas características individuales que pueden condicionar dicho aprendizaje (QUIXADÁ; LINS; TAVARES, 2018).

$\mathrm{Si}$ nos centramos en el primer tipo de trabajos, aquellos que abordan el aprendizaje incidental a través de la lectura, como señala Grabe (2009), existen evidencias científicas de que la lectura extensiva sustenta el aprendizaje del vocabulario, y ello a pesar de las reticencias mostradas por algunos investigadores sobre el escaso aumento del vocabulario a partir de la lectura. Así, según este investigador, la exposición extensiva a la lectura influye en patrones de aprendizaje asociativos que resultan ser la base de las habilidades de lectura y del conocimiento del vocabulario. Pellicer-Sánchez y Schmitt (2010) plantean, por su parte, que, gracias a la lectura extensiva, los estudiantes pueden acceder al vocabulario de frecuencia media que, en ocasiones, resulta complicado aprender, dada la dificultad de abordar en las clases tantos ítems léxicos explícitamente y su exclusión de las lecturas graduadas, que se centran sobre todo en el léxico más frecuente. Asimismo, otros investigadores ponen de manifiesto los beneficios que la lectura puede reportar en el vocabulario. Entre ellos, Santos Díaz (2015) expone que el vocabulario productivo de sus estudiantes, medido a través de una prueba de disponibilidad léxica, así como la identificación de tecnicismos se incrementan conforme lo hace la frecuencia de lectura. $Y$ es que ambos aspectos, vocabulario y lectura, están intimamente ligados, pues, si la lectura facilita el aprendizaje del vocabulario, este favorece el acceso a la lectura.

Santos Díaz (2015) toma en consideración un aspecto clave en nuestro trabajo: la frecuencia de la lectura. Por lo que respecta a los hábitos lectores de los futuros profesores de Educación Primaria, existen pocos trabajos que aborden esta temática cuando el hábito lector hace referencia a una lengua extranjera. Si nos centramos en los hábitos lectores en general, los resultados de las investigaciones no son halagüeños. Granado (2014, p. 44) expone que los futuros maestros son lectores "[...] poco asiduos e inmaduros, frecuentan poca variedad de textos, no dan gran valor a los libros, sobreestiman su práctica lectora, utilizan poco las bibliotecas y hacen un mero uso instrumental de la lectura". Por su parte, Munita (2014) explica que los estudiantes de la Universidad de Barcelona evaluados plantean un perfil definido por trayectorias lectoras discontinuas, intertextos escasos y prácticas de lectura relacionadas mayoritariamente con los best sellers. Vera Valencia (2017), esta vez con estudiantes de Facultades de 
Educación de Castilla la Mancha, confirma la poca continuidad con la que leen los futuros maestros, pues solo un escaso $6 \%$ de su muestra puede ser considerado lector frecuente. En lo que respecta a la lectura en inglés de aquellos estudiantes que se preparan para ser docentes de esta lengua, el trabajo de Gómez López (2014) expone unos resultados que son coincidentes con los anteriores en el sentido de que un elevado porcentaje de su muestra no posee el hábito lector en inglés, leyendo solo aquellos materiales académicos cuya lectura es obligatoria. Asimismo, los análisis efectuados pusieron de manifiesto una fuerte correlación entre el nivel de dominio de inglés y el hábito de lectura, siendo los alumnos de más nivel aquellos que más leen y se acercan a lecturas que no son obligatorias. Santos Díaz (2015), por su parte, en sus análisis con futuros docentes de Secundaria, encuentra que la mayoría de sus encuestados leen algunos libros, pero tan solo el 4,8\% leen más de cinco libros al año. Asimismo, encuentra una correlación positiva significativa entre la frecuencia de lectura en español y en lengua extranjera.

Partiendo de los datos expuestos, en este trabajo pretendemos explorar los hábitos lectores en inglés de los futuros maestros de esta disciplina y relacionarlos con el tamaño de su vocabulario receptivo, que puede ser, además, un indicador de su competencia general en lenguas. Por ello, pretendemos dar respuesta a las siguientes preguntas de investigación: ¿Cuáles son los hábitos lectores de los estudiantes que se preparan para ser futuros docentes de inglés en Educación Primaria?; ¿Cuál es la extensión de su vocabulario receptivo?; ¿Existe una correlación entre la frecuencia de la lectura y el tamaño de su vocabulario receptivo?

\section{Metodología}

La investigación que presentamos se enmarca en un enfoque cuantitativo, con un diseño de tipo transversal, descriptivo correlacional. La muestra es de conveniencia e intencional, aunque se ha contado con el $100 \%$ de la población.

Para el desarrollo de esta investigación, se han utilizado dos instrumentos diferentes que nos han permitido medir el vocabulario receptivo de los estudiantes, el primero, y los hábitos lectores de nuestros estudiantes, el segundo. 
Por lo que respecta al vocabulario receptivo de los estudiantes, hemos recurrido al Vocabulary Level Test (NATION, 1983), utilizando la actualización realizada por Webb, Sasao y Ballance (2017). Se trata de un test de opción múltiple en combinación en el que se le presentan al estudiante 30 preguntas organizadas en diez agrupaciones de seis palabras (tres correctas y tres distractores) y tres definiciones, que debe emparejar. Aunque esta versión se divide en cinco niveles que evalúan el conocimiento de las 1.000 a las 5.000 palabras más frecuentes en inglés, en este estudio nos hemos limitado a los tres primeros. En relación con los hábitos lectores, hemos adaptado el cuestionario de Gómez López (2014), introduciendo, además, preguntas relativas a las lenguas que conocen y los niveles que poseen de ellas.

La muestra de estudio estaba conformada inicialmente por 36 estudiantes que cursan el Itinerario de Intensificación en Lenguas Extranjeras - Inglés - del Grado en Educación Primaria en la Facultad de Educación de la Universidad de Extremadura. Se trata, por tanto, de alumnos del último curso del Grado que se preparan para convertirse en maestros de inglés en esa etapa educativa. Puesto que dos alumnos no completaron todas las pruebas fueron eliminados de la investigación, quedando finalmente la muestra configurada por 34 estudiantes. La media de edad de los sujetos es de 23,5, con una presencia mayoritaria de chicas $(n=20 ; 58,8 \%)$ frente a chicos $(n=14 ; 41,2 \%)$. Todos los estudiantes tienen como lengua materna el español, a excepción de dos informantes cuyas lenguas son el ucraniano y el portugués.

Los datos se obtuvieron al final del primer semestre (febrero de 2020), una vez que había concluido la impartición de las asignaturas teóricas que componen el Itinerario.

En primer lugar, se procedió a contabilizar el número de aciertos obtenidos en cada uno de los niveles de frecuencia analizados y después se estimó el tamaño del léxico, en base a los resultados obtenidos y siguiendo la fórmula establecida por Nation (1990). Para efectuar el análisis estadístico, se ha utilizado el software estadístico Statistical Package for the Social Sciences (SPSS), versión 19. Tras el estudio de los datos, se determinó la idoneidad de las pruebas estadísticas a realizar y se estableció el tamaño del efecto mediante la $d$ de Cohen (1988). 


\section{Resultados y discusión}

En respuesta a la primera pregunta de investigación que interrogaba acerca de los hábitos lectores de los futuros docentes en Educación Primaria, la Figura 1 nos muestra la distribución del alumnado entre aquellos que declaran tener el hábito lector en español y en inglés:

Figura 1 - Hábito lector en lengua materna y extranjera

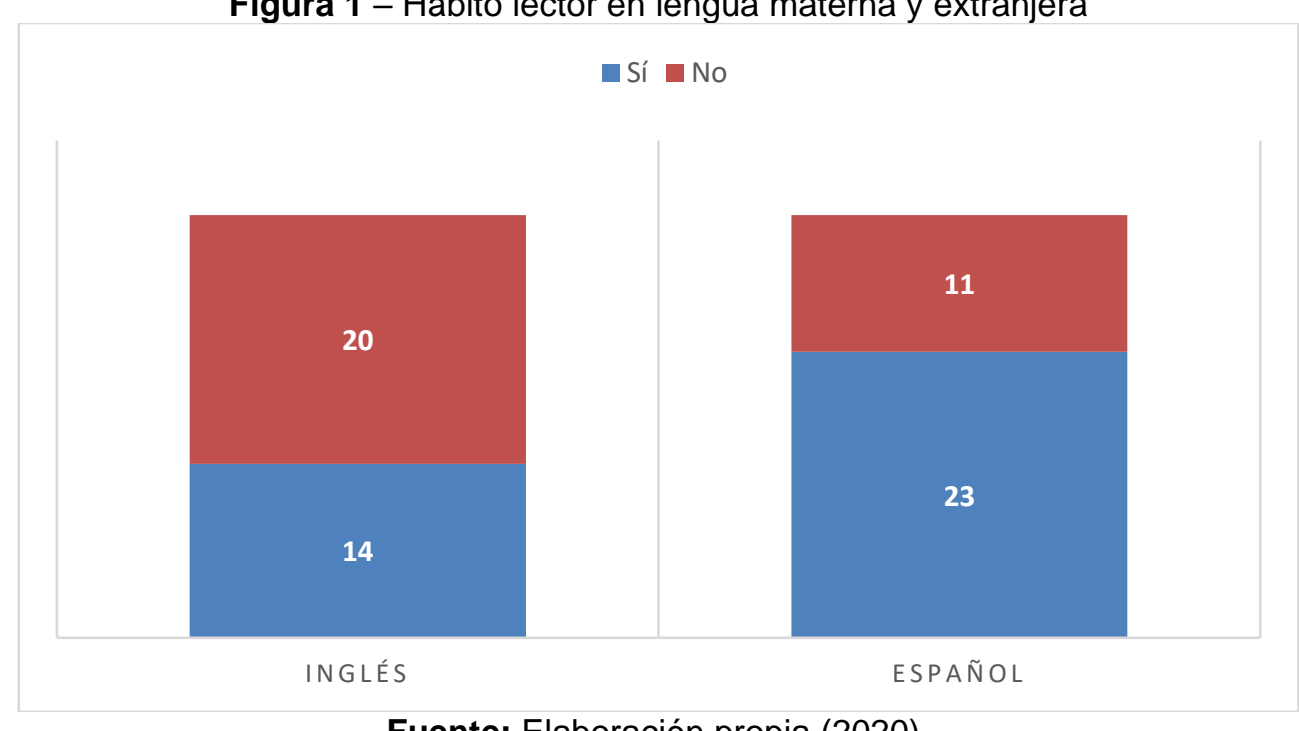

Fuente: Elaboración propia (2020).

Como se observa en la figura, el hábito lector está más consolidado en lengua materna que en la lengua extranjera. Mientras que, en la primera, el $67,7 \%$ de la muestra manifiestan tener dicho hábito desarrollado, en la segunda tan solo lo tienen el $41,2 \%$, mientras que un mayoritario $58,8 \%$ declaran no poseer hábito lector en la lengua que va enseñar. Sin embargo, un dato interesante es que si bien un 56,5\% tienen el hábito lector en español, pero no en inglés, del 71,4 \% de los que leen en inglés habitualmente lo hacen también es español, es decir, que son lectores en ambas lenguas. Este resultado es coincidente con el obtenido por Santos Díaz (2015), pues en su estudio existía una correlación positiva al $99 \%$ entre la frecuencia de lectura en español y en la lengua extranjera. No obstante, si nos centramos específicamente en los datos en inglés, los resultados no son muy buenos, dado que, como hemos comentado, la lectura es una fuente importante de aprendizaje en relación con la lengua. Nuestros resultados son mejores que los obtenidos por Gómez López (2014), aunque es cierto que, en su caso, el cuestionario fue pasado a alumnos tanto del Grado en Educación

Educ. Form., Fortaleza, v. 6, n. 1, e3504, jan./abr. 2021

DOI: https://doi.org/10.25053/redufor.v6i1.3504

https://revistas.uece.br/index.php/redufor/index 
Primaria como del de Infantil y no se centró específicamente en los futuros maestros de inglés.

Por lo que respecta a los materiales leídos, los alumnos, en su mayoría (60,6 \%), manifiestan no limitarse a leer los materiales que se les facilitan en su formación y cuya lectura es aconsejada para aprobar la asignatura. Sobre la longitud de lo que se lee, aunque un buen número no contesta a esta pregunta del cuestionario, el $70 \%$ de los que lo hacen indican que optan por leer textos cortos, que no ocupan más de media página de extensión. Finalmente se les ha preguntado por la frecuencia con la que leen. La Figura 2 presenta cómo se distribuye la muestra teniendo en cuenta dicha cuestión.

Figura 2 - Frecuencia de lectura

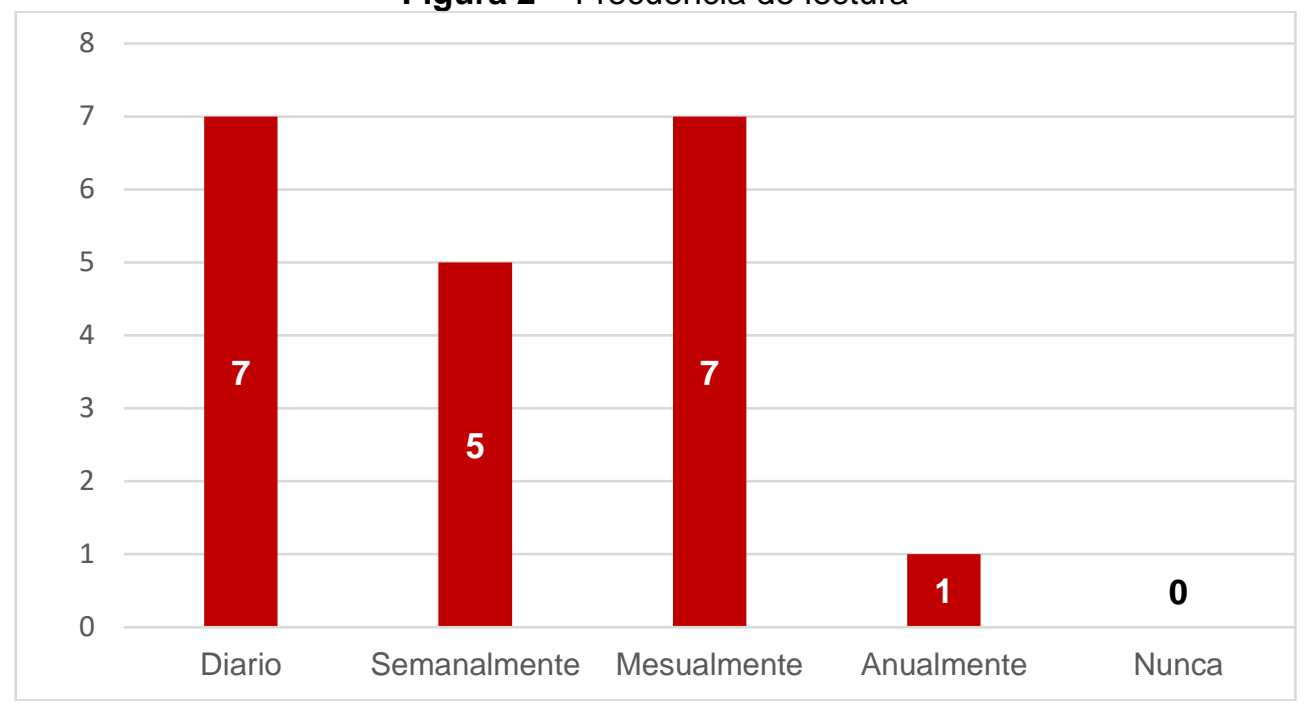

Fuente: Elaboración propia (2020).

Como vemos, un número igual de sujetos realiza algunas lecturas mensualmente, de un lado, y lee a diario, de otro, representando cada caso el $35 \%$ de los que han contestado. Dado que existen 14 casos perdidos, podemos aventurar que se trata de alumnos que no leen nada, de ahí que no hayan contestado, pues esta pregunta estaba destinada solo a los que habían manifestado leer materiales no obligatorios. De ser así, el dato de los que no leen nunca se elevaría al 41,2 \%, cifra que resultaría bastante preocupante, teniendo en cuenta que se trata de futuros profesores de inglés, que pueden encontrar en la lectura una manera de perfeccionar y ampliar sus conocimientos sobre el inglés. En todo caso, en base a los resultados obtenidos, podemos afirmar que la mayor parte de los alumnos lee ocasionalmente, no teniendo muy arraigado el hábito de leer en inglés y ello, a pesar de que un $40 \%$ de la 
muestra se hayan manifestado como lectores habituales. Si comparamos nuestros datos con los obtenidos por Gómez López (2014), encontramos que en nuestra muestra es mayor el porcentaje de los alumnos que leen a diario (35\% frente a un $10 \%$ ) y menor la de los que leen solo en algunas ocasiones a lo largo del año (5\% frente a un $27,5 \%$ ). No obstante, debemos recordar que, aunque las muestras están constituidas por estudiantes de Grados en Educación, la nuestra es específica de alumnos que se preparan para ser docentes de inglés, lo que explicaría los mejores resultados.

Finalmente, por lo que respecta al número de libros leídos, la media es de 1,27 libros al año, repartiéndose la muestra de la siguiente manera:

Figura 3 - Número de libros leídos anualmente en inglés

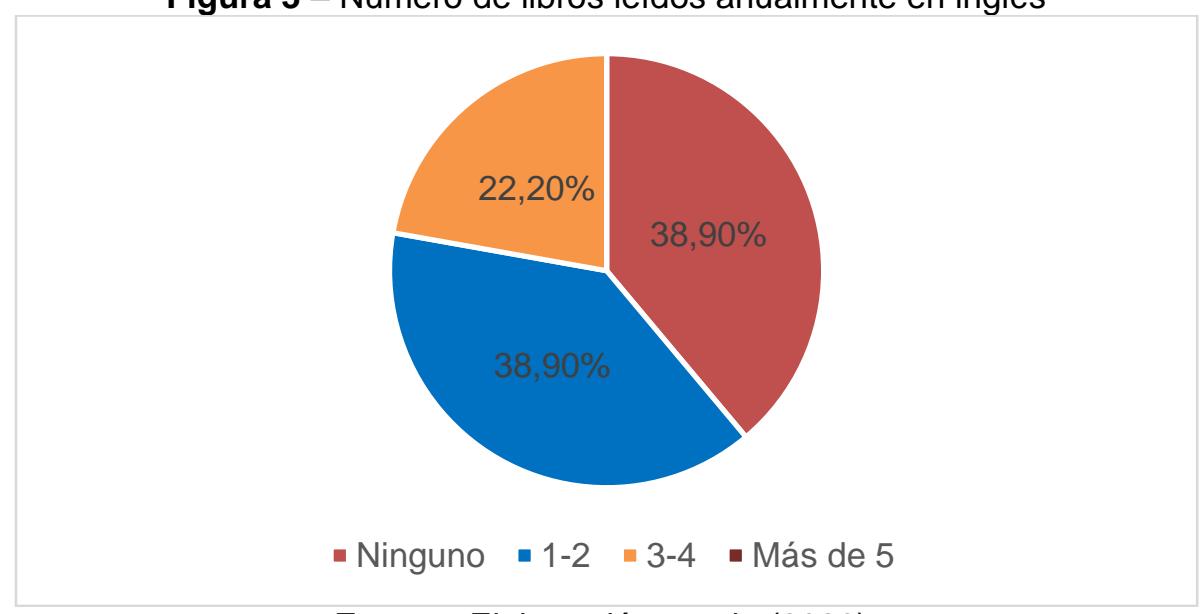

Fuente: Elaboración propia (2020).

La cifra media obtenida, que consideramos insuficiente, pone de nuevo en evidencia la poca importancia que nuestros estudiantes otorgan a la lectura. Sin embargo, si los comparamos con los obtenidos por Santos Díaz (2015) con estudiantes del Máster de Profesorado de Enseñanza Secundaria, nuestros datos resultan en cierta manera coincidentes. Así, en su caso, casi el 50 \% de su muestra no leen ningún libro en inglés, mientras que en el nuestro, si tomamos en consideración los que explícitamente han señalado 0 y los que no han contestado, el porcentaje podría elevarse a un 67,6\%, ampliamente superior al de Santos Díaz (2015). Tanto en nuestro trabajo como en el suyo, la mayoría lee entre uno y cuatro libros, no habiendo en nuestra muestra nadie que lea por encima de esa cifra. 
Nuestra segunda pregunta de investigación pretendía explorar la extensión del vocabulario receptivo. Para ello, se ha evaluado el conocimiento de las tres primeras bandas de frecuencia $(1 \mathrm{~K}, 2 \mathrm{~K}$ y $3 \mathrm{~K})$. La Tabla 1 presenta los descriptivos de los resultados obtenidos.

Tabla 1 - Estadísticos descriptivos $1 \mathrm{~K}, 2 \mathrm{~K}$ y $3 \mathrm{~K}$

\begin{tabular}{cccccc}
\hline & N & Mínimo & Máximo & Media & Desv. típ. \\
\hline $1 \mathrm{~K}$ & 34 & 25 & 30 & 28,50 & 1,638 \\
$2 \mathrm{~K}$ & 34 & 9 & 30 & 24,06 & 4,605 \\
$3 \mathrm{~K}$ & 34 & 0 & 29 & 20,09 & 6,806 \\
N válido (según lista) & 34 & & & \\
\hline \multicolumn{7}{r}{ Fuente: Elaboración propia (2020). }
\end{tabular}

Como se puede observar a partir de los resultados, la media obtenida indica que los alumnos tienen prácticamente dominada la banda de las 1.000 palabras más frecuentes, pues más de la mitad $(61,8 \%)$ ha obtenido una puntuación igual o superior a 29, cifra establecida por los autores de esta versión del Vocabulary Levels Test (VLT) como indicador del dominio de ese léxico. Sin embargo, aunque esas cifras son positivas, no podemos olvidar que 13 alumnos no dominan aún las palabras más frecuentes del inglés de acuerdo con la lista de frecuencia del British National Corpus/Corpus of Contemporary American English (NATION, 2012).

Conforme vamos avanzando hacia el léxico menos frecuente, advertimos cómo desciende el número de alumnos que dominan las bandas de frecuencia superiores. Así, mientras en la $2 \mathrm{~K}$ la media de palabras conocidas es de 24,06 , ya solo hay siete estudiantes que dominan esa banda de frecuencia y el valor mínimo obtenido, situado en nueve palabras, se encuentra muy lejos del dominio (también establecido en 29 palabras) de esas 1.000 palabras. Cuando fijamos nuestra atención en la 3K, vemos cómo la media desciende de nuevo, y tan solo dos estudiantes acreditan dominar las siguientes 1.000 palabras, no habiendo obtenido ninguno el máximo de 30 palabras acertadas.

La Figura 4 nos presenta la estimación de palabras conocidas, según los datos anteriores, utilizando la fórmula de Nation (1990) para la conversión del número de aciertos en palabras. 
Figura 4 - Extensión del vocabulario en las distintas bandas de frecuencia

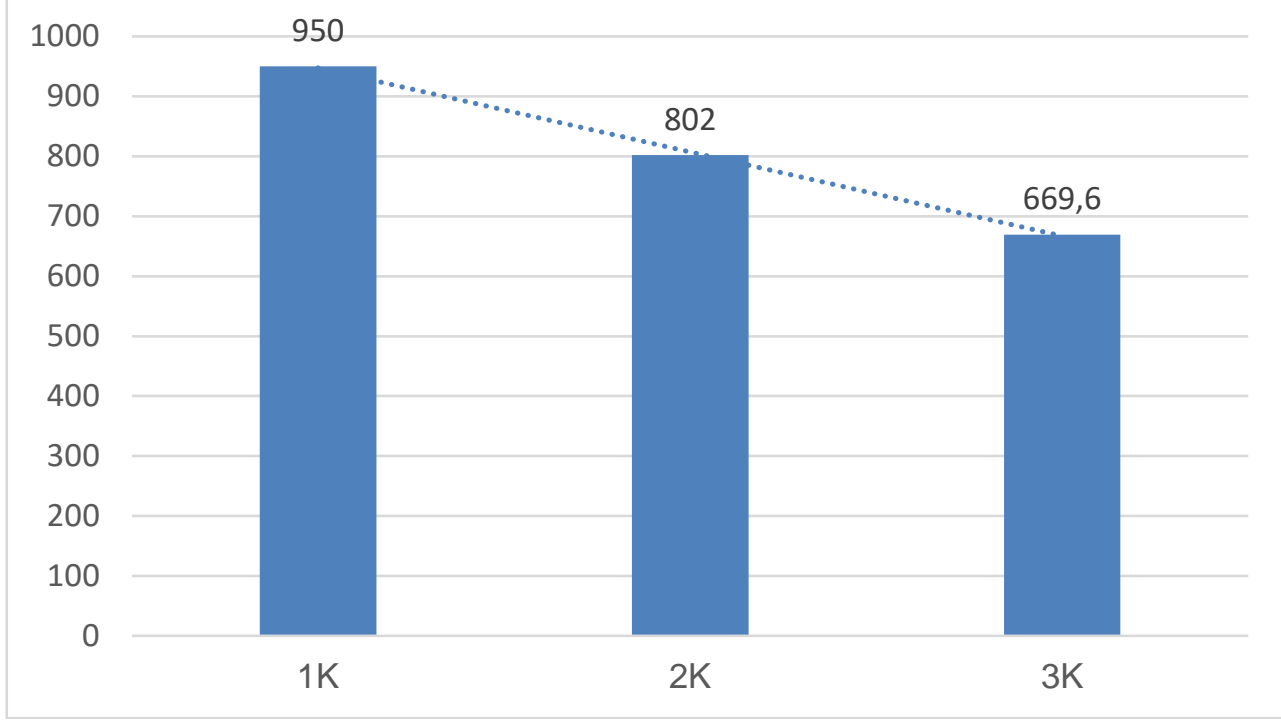

Fuente: Elaboración propia (2020).

Como ya exponíamos anteriormente, mientras en la primera banda de frecuencia los alumnos están, de media, muy cerca de conocer las 1.000 palabras que la conforman, el conocimiento va decreciendo conforme va aumentando el carácter infrecuente del vocabulario. La línea de tendencia muestra el perfil de frecuencia típico planteado por Meara (1992), en el que el trazo desciende de izquierda a derecha, reflejando que los estudiantes de una lengua extranjera conocen más vocabulario frecuente y que este conocimiento disminuye cuando el vocabulario es cada vez menos frecuente.

La Tabla 2, por su parte, presenta los estadísticos descriptivos de la estimación del número de palabras conocidas.

Tabla 2 - Estadísticos descriptivos de las palabras conocidas

\begin{tabular}{lccccc}
\hline & N & Mínimo & Máximo & Media & Desv. típ. \\
\hline Palabras conocidas & 34 & 1366,67 & 2966,67 & 2421,5697 & 378,98298 \\
N válido (según lista) & 34 & & & & \\
\hline
\end{tabular}

Fuente: Elaboración propia (2020).

Como ya hemos comentado anteriormente, tomando en consideración las bandas de frecuencia individualmente, siguiendo las indicaciones de Webb, Sasao y Ballance (2017), son muy pocos los alumnos que podemos considerar que han dominado el léxico comprendido en todas ellas, dos estudiantes concretamente y siete, si contemplamos solo las dos primeras. No obstante, si estudiamos la media de palabras conocidas globalmente 
de entre las 3.000 más frecuentes, la cifra asciende a 2.421, aunque, como muestra la desviación típica, existen importantes variaciones entre ellos. Si atendemos a los umbrales léxicos que facilitan la realización de ciertas actividades comunicativas, el conocimiento medio de vocabulario que presenta la muestra no habilitaría aún para alcanzar una cobertura del $95 \%$ que permitiría comprender oralmente de manera adecuada, según las estimaciones de Van Zeeland y Schmitt (2013), ni tampoco el umbral léxico mínimo fijado por Laufer y Ravenhorst-Kalovski (2010) para la lectura independiente, lo que puede explicar que la muestra no tenga aún adquirido el hábito de lectura, pues desconocen el léxico necesario para una lectura que no requiere de ninguna ayuda.

No podemos contrastar estos resultados con otros de estudiantes de las mismas características que nuestra muestra, es decir, futuros maestros de inglés en Educación Primaria, ya que no conocemos trabajos que se hayan interesado por esa cuestión. Los estudios realizados en el contexto español se han centrado más en explorar el vocabulario receptivo de los estudiantes de últimos cursos de Educación Primaria y Secundaria. Así, por ejemplo, Canga Alonso (2013) se centra en estudiantes de $4^{\circ}$ de la Educación Secundaria Obligatoria (ESO) que presentan un vocabulario receptivo, medido también con el VLT, aunque en la versión de Schmitt, N., Schmitt, D. y Clapham (2001), de 935,33 palabras de media. Dos años de Bachillerato y tres años de estudios universitarios separan unos y otros, pero el aumento de vocabulario es bastante considerable. También resulta mayor el vocabulario de nuestra muestra que el de los estudiantes de Escuela Oficial de Idiomas estudiados por Canga Alonso y Fernández Fontecha (2014), que conocen una media de 1.658 palabras al finalizar el segundo año de estudios.

Finalmente la tercera pregunta de investigación tiene como objetivo determinar si existe correlación alguna entre la frecuencia de la lectura y el tamaño de su vocabulario receptivo. En primer lugar, hemos comparado el tamaño del vocabulario entre los alumnos que declaran poseer el hábito lector en inglés y aquellos que no lo tienen adquirido. Puesto que no se cumplía la homogeneidad de la varianza, hemos aplicado la prueba $U$ de Mann-Whitney, cuyo resultado $(Z=-, 298 ; p=, 766)$ nos informa de que no existen diferencias en la extensión del vocabulario en función de si se posee o no hábito lector en inglés. Asimismo, si interpretamos la magnitud del tamaño del efecto (LENHARD, W.; LENHARD, A., 2016), observamos cómo, en este caso, es inexistente 
$(d=, 102)$. Seguidamente hemos procedido a realizar un análisis de correlación entre la frecuencia de lectura y el tamaño del vocabulario, para lo que hemos aplicado la prueba no paramétrica de Spearman. En este análisis nos hemos centrado en los 20 alumnos que han respondido a esta pregunta, puesto que los 14 que habían manifestado no poseer hábito lector no han contestado. Los resultados nos indican que no existe relación estadísticamente significativa entre la frecuencia de lectura y el vocabulario receptivo $(r=, 005, p=, 984)$. De este modo, podemos concluir que los alumnos que leen a diario 0 semanalmente no obtienen mejores resultados en el test $y$, por lo tanto, no tienen un vocabulario receptivo mayor que aquellos alumnos que leen en varias ocasiones al mes 0 al año. Aunque existe la creencia de que un mayor vocabulario puede estar relacionado con una lectura frecuente, nuestros resultados contradicen esa idea. Santos Díaz (2015) sí encuentra una correlación entre la frecuencia de la lectura y el vocabulario en las dos lenguas extranjeras analizadas, aunque, en su caso, es el vocabulario productivo, medido a través de una prueba de disponibilidad, el que es objeto de análisis. Curiosamente, es en lengua materna donde esa relación no está presente.

\section{Conclusiones}

En este trabajo hemos pretendido indagar en la relación existente entre los hábitos lectores de una muestra constituida por futuros profesores de inglés y su vocabulario receptivo. Las respuestas a las preguntas de investigación formuladas nos permiten concluir lo siguiente:

- Los estudiantes que constituyen la muestra no tienen consolidado el hábito lector, que está mucho más presente en lengua materna que en lengua extranjera. Pocos son los alumnos que leen a diario y un elevado porcentaje no se acerca de ningún modo a textos escritos en la lengua que tienen pensado enseñar. Por lo tanto, sería interesante, especialmente para los futuros profesores de inglés, animar, de un lado, a la lectura de textos que les van a permitir ampliar sus conocimientos, fomentando la lectura de imágenes, $u$ otro tipo de lenguaje no verbal que acompañe los textos escritos (NÓBREGA, 2016) y, de otro, indagar en los motivos por los que no hacen de la lectura de textos en inglés una actividad cotidiana. 
- El vocabulario receptivo no alcanza las 3.000 palabras más frecuentes, lo que plantea problemas a la hora de comprender textos orales y escritos. Este puede ser el motivo por el que los alumnos tienen dificultades para leer; de ahí que sea necesario profundizar en el conocimiento del léxico más frecuente, pues, como señalan Milton y Treffers-Daller (2013), el tamaño del vocabulario está claramente relacionado con la adquisición de la competencia de lectura.

- No existe una relación entre la frecuencia de la lectura y el vocabulario receptivo de los estudiantes analizados.

Nuestro trabajo presenta algunas limitaciones en relación con la muestra. A pesar de haber trabajado con el total de alumnos que cursan el Itinerario de Intensificación en Lenguas Extranjeras - inglés -, el número total no es excesivamente amplio. Se abre a este respecto la necesidad de replicar el estudio con nuevos estudiantes, lo que nos permitirá obtener más datos que faculten analizar si se produce o no la confirmación de los resultados aquí obtenidos. Además, sería conveniente indagar con mayor profundidad en los hábitos lectores de nuestros alumnos y especialmente en las causas que motivan la ausencia de dicho hábito lector.

Como hemos expuesto anteriormente, el tamaño del léxico de los estudiantes que se preparan para ser profesores de lengua inglesa así como sus hábitos lectores no han sido cuestiones muy analizadas, de ahí que no se puedan establecer comparaciones con otros estudios. A este respecto, creemos necesario continuar trabajando en esta línea de investigación y pensamos que, como complemento al análisis aquí desarrollado, se puede igualmente estudiar la relación entre la frecuencia de lectura y el vocabulario, considerado en su vertiente productiva.

\section{Referencias}

ADOLPHS, S.; SCHMITT, N. Vocabulary coverage according to spoken discourse context. In: BOGAARDS, P.; LAUFER, B. (ed.). Vocabulary in a second language. Amsterdam: John Benjamins, 2004. p. 39-49.

AGUSTÍN LLACH, M. P.; TERRAZAS GALLEGO, M. Examining the relationship between receptive vocabulary size and written skills of primary school learners. Atlantis, v. 31, n. 1, p. 129-147, 2009. 
ALDERSON, J. C. Diagnosing foreign language proficiency: the interface between learning and assessment. London: Continuum, 2005.

BERTAUX, P. et al. The CLIL Teacher's Competence Grid. 2010. Disponible en: http://tplusm.net/clil_competences_grid_31.12.09.pdf. Accedido el: 7 mar. 2018.

BLEICHENBACHER, L. et al. Teacher competences for languages in education: conclusions of the project. Graz/Strasbourg, 2016.

BOGAARDS, P. Le vocabulaire dans l'apprentissage des langues étrangères. Paris: Didier, 1994.

CANGA ALONSO, A. Receptive vocabulary size of secondary Spanish EFL learners. Revista de Lingüística y Lenguas Aplicadas, Valencia, v. 8, p. 66-75, 2013.

CANGA ALONSO, A.; FERNÁNDEZ FONTECHA, A. Motivation and L2 receptive vocabulary knowledge of Spanish EFL learners at the Official School of Languages. Miscelánea, Zaragoza, v. 49, p. 13-28, 2014.

COHEN, J. Statistical power analysis for the behavioral sciences. 2. ed. Hillsdale: Erlbaum, 1988.

FAN, M. How big is the gap and how to narrow it? An investigation into the active and passive vocabulary knowledge of $L 2$ learners. RELC Journal, London, v. 31, n. 2, p. 105$119,2000$.

FRIGOLS, M. J. et al. European Framework for CLIL. Graz: The European Center for Modern Language, 2011.

GÓMEZ LÓPEZ, Á. Los hábitos lectores en inglés de futuros maestros: implicaciones didácticas. Investigaciones sobre Lectura, Málaga, n. 2, p. 24-31, 2014.

GRABE, W. Reading in a second language. New York: Cambridge University, 2009.

GRANADO, C. Teachers as readers: a study of the reading habits of future teachers. Culture and Education, v. 26, n. 1, p. 44-70, 2014.

HILTON, H. La compétence lexicale en production orale. In: DILTEC, 2007, Paris. Actes... Paris: Université Paris 3, 2007. p. 1-10.

HILTON, $\mathrm{H}$. The link between vocabulary knowledge and spoken L2 fluency. The Language Learning Journal, v. 36, n. 2, p. 153-166, 2008.

HORST, M.; COBB, T.; MEARA, P. Beyond a clockwork orange: acquiring second language vocabulary trough reading. Reading in a Foreign Language, v. 11, n. 2, p. 207223, 1998. 
LAUFER, B. How much lexis is necessary for reading comprehension?. In: ARNAUD, P.; BÉJOINT, H. (ed.). Vocabulary and applied linguistics. London: Macmillan, 1992. p. 126-132.

LAUFER, B. Possible changes in attitudes towards vocabulary acquisition research. IRAL, v. 24, p. 69-75, 1986.

LAUFER, B. What percentage of text-lexis is essential for comprenhension?. In: LAUREN, C.; NORDMAN, M. (ed.). Special language: from humans thinking to thinking machines. Clevedon: Multilingual Matters, 1989. p. 316-323.

LAUFER, B.; RAVENHORST-KALOVSKI, G. G. Lexical threshold revisited: lexical text coverage, learners' vocabulary size and reading comprehension. Reading in a Foreign Language, v. 22, n. 1, p. 15-30, 2010.

LENHARD, W.; LENHARD, A. Calculation of effect sizes. Dettelbach: Psychometrica, 2016.

MEARA, P. EFL Vocabulary Tests. Swansea: Centre for Applied Language Studies, 1992.

MEARA, P.; JONES, G. Vocabulary size as a placement indicator. Applied Linguistics in Society, p. 80-87, 1988.

MILTON, J. The development of vocabulary breadth across the CEFR levels. In: BARTNING, I.; MARTIN, M.; VEDDER, I. (ed.). Communicative proficiency and linguistic development: intersections between SLA and language testing research. [S.I.]: Eurosla, 2010. p. 211-232.

MILTON, J.; TREFFERS-DALLER, J. Vocabulary size revisited: the link between vocabulary size and academic achievement. Applied Linguistics Review, v. 4, n. 1, p. 151-172, 2013.

MILTON, J.; WADE, J.; HOPKINS, N. Aural word recognition and oral competence in a foreign language. In: CHACÓN, R.; ABELLO, C.; TORREBLANCA, M. (ed.). Insigths into non-native vocabulary teaching and learning. Clevedon: Multilingual Matters, 2010. p. 83-98.

MONTERO-PEREZ, M. et al. Effects of captioning on video comprehension and incidental vocabulary learning. Language Learning \& Technology, v. 18, p. 118-141, 2014.

MONTERO-PEREZ, M. Incidental vocabulary learning through viewing video. Studies in Second Language Acquisition, p. 1-25, 2019.

MUNITA, F. Reading habits of pre-service teachers. Culture and Education, v. 26, n. 3, p. 448-475, 2014.

NATION, P. Learning vocabulary in another language. Cambridge: Cambridge University, 2001.

NATION, P. Teaching and learning vocabulary. New York: Newbury House, 1990.

Educ. Form., Fortaleza, v. 6, n. 1, e3504, jan./abr. 2021

DOI: https://doi.org/10.25053/redufor.v6i1.3504

https://revistas.uece.br/index.php/redufor/index 
NATION, P. Testing and teaching vocabulary. Guidelines, v. 5, n. 1, p. 12-25, 1983.

NÓBREGA, D. As tirinhas nas aulas de leitura em língua inglesa: uma proposta didáticopedagógica para iniciantes. Educação \& Formação, Fortaleza, v. 1, n. 3, p. 154-167, 2016.

OVTCHAROV, V.; COBB, T.; HALTER, R. La richesse lexicale des productions orales: Mesure fiable du niveau de compétence langagière. The Canadian Modern Language Review/La revue canadienne des langues vivantes, 2006.

PAVÓN, V.; ELLISON, M. Examining teacher roles and competences in Content and Language Integrated Learning (CLIL). Linguarum Arena, v. 4, p. 65-78, 2013.

PELLICER-SÁNCHEZ, A. Learning L2 collocations incidentally from reading. Language Teaching Research, v. 21, n. 3, p. 381-402, 2017.

PELLICER SÁNCHEZ, A.; SCHMITT, N. Incidental vocabulary acquisition from an authentic novel: do things fall apart?. Reading in a Foreign Language, v. 22, n. 1, p. 31-55, 2010.

PÉREZ CAÑADO, M. L. Are teachers ready for CLIL? Evidence from a European study. European Journal of Teacher Education, v. 39, p. 202-221, 2016.

PETERS, E.; WEBB, S. Incidental vocabulary acquistion through viewing L2 television and factors that affect learning. Studies in Second Language Acquisition, v. 40, n. 3 , p. 551-577, 2018.

QIAN, D. D. Investigating the relationship between vocabulary knowledge and academic reading performance: an assessment perspective. Language Learning, v. 52, n. 3, p. 513-536, 2002.

QUIXADÁ, L. M.; LINS, S. G.; TAVARES, A. C. O lúdico como atividade discursiva e como uma via para a formaçao do leitor: teoria e relato de pesquisa em uma escola pública em Fortaleza-CE. Educação \& Formação, Fortaleza, v. 3, n. 7, p. 182-199, 2018.

RESTREPO RAMOS, F. D. Incidental vocabulary learning in second language acquisition: a literature review. Profile Issues in Teachers' Professional Development, v. 17 , n. 1, p. 157-166, 2015.

RICHARDS, J. C. The role of vocabulary teaching. Tesol Quarterly, v. 10, n. 1, p. 77-89, 1976.

RODGERS, M. P. H.; WEBB, S. Incidental vocabulary learning through viewing television. ITL, 2019.

SANTOS DÍAZ, I. C. Incidencia de la lectura en el vocabulario en lengua materna y extranjera. Ocnos, Niterói, v. 16, n. 1, p. 79-88, 2015.

SCHMITT, N. Researching vocabulary. London: Palgrave Macmillan, 2010. 
SCHMITT, N.; SCHMITT, D.; CLAPHAM, C. Developing and exploring the behaviour of two new versions of the Vocabulary Level Test. Language Testing, v. 18, p. 58-88, 2001.

STAEHR, L. S. Vocabulary size and the skills of listening, reading and writing. The Language Learning Journal, v. 36, n. 2, p. 139-152, 2008.

VAN ZEELAND, H.; SCHMITT, N. Lexical coverage in L1 and L2 listening comprehension: the same or different from reading comprehension?. Applied Linguistics, v. 34, n. 4, p. 457479, 2013.

VERA VALENCIA, S. Reading motivation and reading habits of future teachers. Investigaciones sobre Lectura, v. 7, p. 85-96, 2017.

WANG, Y.; TREFFERS-DALLER, J. Explaining listening comprehension among L2 learners of English: the contribution of general language proficiency, vocabulary knowledge and metacognitive awareness. System, v. 65, 2017.

WEBB, S.; CHANG, A. C.-S. Second language vocabulary growth. RELC Journal, v. 43, n. 1, p. 113-126, 2012.

WEBB, S.; CHANG, A. C.-S. Second language vocabulary learning through extensive reading with audio support: how do frequency and distribution of occurrence affect learning?. Language Teaching Research, v. 19, n. 6, p. 667-686, 2015.

WEBB, S.; NATION, P. How vocabulary is learned. Oxford: Oxford University, 2017.

WEBB, S.; SASAO, Y.; BALLANCE, O. The updated vocabulary levels test. ITL, v. 168, n. 1, p. 33-69, 2017.

WILKINS, D. A. Linguistics in language teaching. London: Edward Arnold, 1974.

ZAREVA, A.; SCHWANENFLUGEL, P.; NIKOLOVA, Y. Relationship between lexical competence and language proficiency variable sensitivity. Studies in Second Language Acquisition, v. 27, p. 567-595, 2005.

\footnotetext{
Guadalupe de la Maya Retamar, Universidad de Extremadura, Departamento de Didáctica de las Ciencias Sociales, de las Lenguas y las Literaturas

i(D)http://orcid.org/0000-0003-4201-4024

Profesora titular de Universidad del Departamento de Didáctica de las Ciencias Sociales, de las Lenguas y las Literaturas de la Universidad de Extremadura. Imparte docencia en el Grado de Educación Primaria y en el Máster para la enseñanza bilingüe en Educación Primaria y Secundaria. Sus principales líneas de investigación se centran en la formación de profesorado de lenguas extranjeras, adquisición del vocabulario y aprendizaje de terceras lenguas y lenguas adicionales.

Contribución de autoría: Administración del proyecto, análisis formal, concepto, curaduría de datos, redacción.

E-mail: gmaya@unex.es
} 
Magdalena López-Pérez, Universidad de Extremadura, Departamento de Didáctica de las Ciencias Sociales, de las Lenguas y las Literaturas

ii(D)https://orcid.org/0000-0001-6233-1719

Profesora contratada doctora del Departamento de Didáctica de las Ciencias Sociales, de las Lenguas y las Literaturas de la Universidad de Extremadura. Imparte docencia en los Grados de Educación Infantil y Educación Primaria y en el Máster para la enseñanza bilingüe en Educación Primaria y Secundaria. Sus principales líneas de investigación se centran, por un lado, en el análisis de las metodologías y estrategias para la enseñanza y el aprendizaje de la lengua extranjera (inglés) incidiendo en cómo las emociones pueden afectar a dicho proceso y, por otro lado, en la aplicación de recursos TIC para el aprendizaje de la lengua inglesa y para la educación bilingüe, desde un enfoque práctico e interdisciplinar.

Contribución de autoría: Primera redacción, redacción - revisión y edición, investigación, metodología, financiación, recursos, software, supervisión, validación y visualización.

E-mail:magdalenalopez@unex.es

Editora responsable: Lia Machado Fiuza Fialho Pareceristas ad hoc: María-Pilar Molina-Torres y María Victoria López Pérez

\section{Cómo citar este artículo (ABNT):}

MAYA RETAMAR, Guadalupe de la; LÓPEZ-PÉREZ, Magdalena. Hábitos lectores y competencia léxica de futuros profesores de Inglés en educación primaria. Educ. Form., Fortaleza, v. 6, n. 1, e3504, 2020. Disponible en:

https://revistas.uece.br/index.php/redufor/article/view/3504

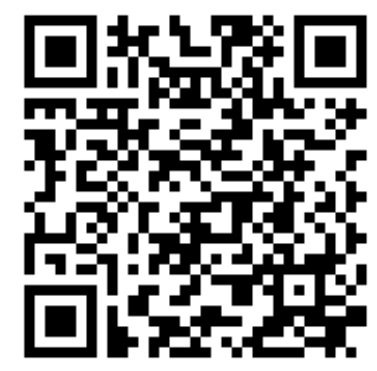

Recibido el 9 de julio de 2020.

Aceptado el 6 de agosto de 2020.

Publicado el 12 de noviembre de 2020.

Educ. Form., Fortaleza, v. 6, n. 1, e3504, jan./abr. 2021

DOI: https://doi.org/10.25053/redufor.v6i1.3504

https://revistas.uece.br/index.php/redufor/index 